\title{
Fms-like tyrosine kinase 3 ligand is required for thymic dendritic cell generation from bone marrow-derived $\mathrm{CD}_{117^{+}}$hematopoietic progenitor cells
}

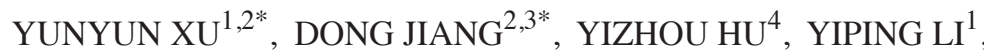 \\ XUEGUANG ZHANG ${ }^{2}$, JIAN WANG ${ }^{1}$ and YONG WANG ${ }^{5}$
}

\begin{abstract}
${ }^{1}$ Institute of Pediatrics, Children's Hospital Affiliated to Soochow University Suzhou, Jiangsu 215025;
${ }^{2}$ Stem Cell and Biomedical Material Key Laboratory of Jiangsu Province, State Key Laboratory Incubation Base, Soochow University; ${ }^{3}$ Department of Cardiothoracic Surgery, The First Affiliated Hospital of Soochow University, Suzhou, Jiangsu 215007, P.R. China; ${ }^{4}$ Department of Virology, The Haartman Institute,

Molecular Cancer Biology Research Program and Helsinki University Hospital, University of Helsinki, Helsinki FIN-00100, Finland; ${ }^{5}$ Department of Medicine, Division of Pulmonary Allergy and Critical Care Medicine, University of Alabama, Birmingham, AL 35233, USA
\end{abstract}

Received October 8, 2014; Accepted August 17, 2015

DOI: $10.3892 / \mathrm{mmr} .2015 .4320$

\begin{abstract}
Thymic dendritic cells (TDCs) are a type of dendritic cell (DC) in the thymus, which can enhance the proliferation of thymic $\mathrm{T}$ lymphocytes, regulate negative selection and induce central tolerance through autoantigen presentation. However, further investigations using TDCs has been restricted due to insufficient numbers. Therefore, an effective expansion method for TDCs in vitro is urgently required to further examine their biological characteristics. In the present study, a novel system was established using fetal thymus organ culture (FTOC) and a hanging drop culture system in the presence of fms-like tyrosine kinase 3 ligand (Flt3L), termed the Flt3L/FTOC system. TDCs were successfully generated and expanded from $\mathrm{CD} 117^{+}$bone marrow hematopoietic progenitor cells. Conventional DCs (cDCs; CD11 $\left.{ }^{+} \mathrm{B} 220^{-} \mathrm{DCs}\right)$ and plasmacytoid DCs (pDCs; CD11 $\left.\mathrm{c}^{+} \mathrm{B} 220^{+} \mathrm{DCs}\right)$ were found in the TDCs generated using the Flt3L/FTOC system. These cells exhibited the specific morphological features of DCs, which were confirmed using Giemsa staining. Furthermore, the cytokine and surface marker profiles were also analyzed.
\end{abstract}

Correspondence to: Professor Yong Wang, Department of Medicine, Division of Pulmonary Allergy and Critical Care Medicine, University of Alabama, 1900 University Boulevard, Birmingham, AL 35233, USA

E-mail: yongw@uab.edu

${ }^{*}$ Contributed equally

Key words: Fms-like tyrosine kinase 3 ligand, fetal thymus organ culture, $\mathrm{CD} 117^{+}$hematopoietic progenitor cells, thymic dendritic cells
Higher expression levels of interferon- $\alpha$ and interleukin-12 were observed in the pDCs, compared with the cDCs, and higher expression levels of toll-like receptor (TLR)7 and TLR9 were found in the pDCs than in the cDCs. In addition, the Flt3L/FTOC-derived TDCs also exhibited the ability to stimulate the allogenic $\mathrm{T}$ cell response. In conclusion, a novel in vitro culture system of thymic $\mathrm{cDCs}$ and $\mathrm{pDCs}$ using Flt3L was established, and this may provide a methodological basis for understanding the properties of TDCs.

\section{Introduction}

Dendritic cells (DCs) include a range of subtypes, which can induce $\mathrm{T}$ cell immune responses, and are the key regulators of the human immune system $(1,2)$. However, the origin of DCs and the growth factor requirements during their development remain to be fully elucidated. Studies have demonstrated that substantial numbers of functional DCs can be obtained from the peripheral blood or bone marrow (BM) precursor cells through the effects of different cytokines $(3,4)$. Additionally, DCs can also arise from intrathymic progenitor cells, which are able to form $\mathrm{T}$ lymphocytes $(5,6)$. In the mouse, at least two DC subpopulations have been identified in the thymus, based on the expression of CD11c and B220, which are termed conventional DCs (cDCs; CD11 $\left.\mathrm{c}^{+} \mathrm{B} 220^{-} \mathrm{DCs}\right)$ and plasmacytoid DCs (pDCs; CD11 $\mathrm{c}^{+} \mathrm{B} 220^{+} \mathrm{DCs}$ ), respectively. Among these, cDCs exhibit a high capacity for antigen processing and presentation through major histocompatibility complex class I and II molecules to T cells (7). In addition pDCs are potent type I interferon (IFN) producers (7-9), which are critical in protecting the thymus against viral infection $(10,11)$ and thymocytes positive selection through the secretion of IFN- $\alpha(12,13)$. However, the exact properties of thymic DCs (TDCs) remain to be fully elucidated due to the current limitations in the expansion method in vitro. 
In previous years, considerable efforts have been made to improve this. Varas et al (14) demonstrated that interleukin (IL)-7 can increase the production of DCs using fetal thymus organ culture (FTOC).

Fms-like tyrosine kinase 3 ligand (Flt3L), also termed fetal liver kinase-2, is a receptor tyrosine kinase expressed on hematopoietic stem cells and early precursors (15). Flt3L represents a key factor in Type I IFN-producing cell (pDC precursor) differentiation. Studies have demonstrated that Flt3L treatment can significantly increase the number of CD11c ${ }^{+}$myeloid DCs and type I IFN-producing cells (16-18). The Flt3L pathway is, therefore, critical in DC homeostasis. However, few studies have focused on the effect of Flt3L in DC differentiation and development in the thymus. In the present study, FTOC was used to imitate the thymic microenvironment, in which TDCs are required. Combined with the method of hanging drop culture, the effect of Flt3L on the differentiation of BM-derived hematopoietic progenitor cells (HPCs) into DCs in the thymus was further examined. The present study hypothesized that a large number of cDCs and pDCs may be routinely generated from a small number of $\mathrm{CD} 117^{+}$HPCs by adding Flt3L. The investigation aimed to provide novel insight into the differentiation and development of TDCs, which may have significant potential in translational medicine.

\section{Materials and methods}

Mice. C57BL/6 mice and BALB/c mice were purchased from the Experimental Animal Center of the Chinese Academy of Sciences (Shanghai, China). In all experiments, 8-12 week old mice (25-30 g) were used. A total of 40 male mice and 60 female mice were used. All mice were bred in the animal facility of the Institute of Medical Biotechnology (Jiangsu, China) under pathogen-free conditions (18-22 $2^{\circ} \mathrm{C} /$ lighting 10-14 h with pelleted food). The present study was approved by the Ethics Committee of Soochow University (Suzhou, China).All methods were performed in accordance with the approved guidelines and experimental protocols, approved by the Ethical Review Board of Soochow University, and the mice used in the present study were handled in strict accordance with recomended animal practices. The day of identification of the vaginal plug was designated as day 0 of gestation. Mouse fetuses at day 15 of gestation were obtained from timed pregnancies.

FTOC. The thymic lobes were aseptically removed from 15-day-old mouse fetuses using a stereoscopic microscope (Olympus SZX7; Olympus Corporation, Tokyo, Japan). The lobes were trimmed of surrounding mesenchyme and were organ-cultured as follows: A total of 4-6 individual thymic lobes were placed on the surface of $0.8-\mu \mathrm{m}$ polycarbonate filters (EMD Millipore, Madrid, Spain), which rested on stainless steel screen sections attached to the central well of organ tissue culture dishes (BD Biosciences, San Diego, CA, USA). The lobes were cultured in $2 \mathrm{ml}$ RPMI 1640 medium supplemented with sodium pyruvate $(1 \mathrm{mmol} / \mathrm{l})$, streptomycin $(100 \mathrm{mg} / \mathrm{ml})$ and penicillin $(100 \mathrm{U} / \mathrm{ml}$; all purchased from Invitrogen Life Technologies, Carlsbad, CA, USA), and $10 \%$ fetal bovine serum (FBS; Gibco Life Technologies,
Carlsbad, CA, USA). FTOC medium was also added with 2-deoxyguanosine (2-dGuo; Sigma-Aldrich, St. Louis, MO, USA), which was used to remove thymocytes within the embryonic thymic lobes. The organ cultures were treated at a concentration of $1.35 \mathrm{mmol} / 12$-dGuo. The peripheral well of the culture dishes was filled with $2 \mathrm{ml}$ sterile distilled water. The cultures were incubated at $37^{\circ} \mathrm{C}$ in a humidified incubator containing $5 \% \mathrm{CO}_{2}$, and the medium was replaced daily. The cultures were incubated at $37^{\circ} \mathrm{C}$ for 5-7 days in a humidified incubator containing $5 \% \mathrm{CO}_{2}$, and the medium was replaced every 2 days.

HPC purification and hanging drop culture. A total of six male C57BL/6 mice were sacrificed by cervical dislocation. The BM cells from the 12 femurs and tibias of the sacrificed mice were collected and centrifuged using Ficoll-Paque centrifugation at $450 \mathrm{~g}$ for $20 \mathrm{~min}$ to pellet granulocytes and erythrocytes at $25^{\circ} \mathrm{C}$. The BM monocytes $\left(6-10 \times 10^{6}\right)$ were washed twice with phosphate-buffered saline (PBS).The HPCs were purified via magnetic-activated cell sorting (MACS) using a MiniMacs equipment (Miltenyi Biotec GmbH, Teterow, Germany). The cells were pelleted and incubated with anti-CD117 phycoerythrin (PE)-conjugated cross-reactive rat anti-mouse monoclonal antibodies (clone $3 \mathrm{C} 11 ; 20 \mu 1 / 10^{7}$ cells; Miltenyi Biotec $\mathrm{GmbH}$ ) in the presence of $0.5 \%$ FBS in PBS at $4^{\circ} \mathrm{C}$ for $15 \mathrm{~min}$. The marked cells were washed with PBS and incubated with anti-phycoerythrin (PE) microbeads (100 $\mu \mathrm{l} / 10^{7}$ cells; Miltenyi Biotec Inc, Auburn, CA, USA) in the presence of $0.5 \% \mathrm{FBS}$ in $\mathrm{PBS}$ at $4^{\circ} \mathrm{C}$ for $15 \mathrm{~min}$. The cells were washed, resuspended and purified via MACS. The purity of the sorted cells was determined by re-analyzing a small sample of collected cells and a purity of $>95 \%$ was observed. The sorted CD $117^{+} \mathrm{HPCs}$ and embryonic thymic lobes from the 2-dGuo-treated FTOC system were seeded in a Terasaki plate (Sumitomo Bakelite Co., Ltd., Tokyo, Japan). Subsequently, the plate was turned over and incubated for $48 \mathrm{~h}$ at $37^{\circ} \mathrm{C}$ in a humidified incubator containing $5 \% \mathrm{CO}_{2}$. After $48 \mathrm{~h}$ culture, the lobes in each well were collected and cultured using the FTOC system. For following experiments, the lobes were divided into two groups, consisting of a control group and the Flt3L group. In the control group, the thymic lobes were cultured with complete RPMI 1640 media (20 mmol/l HEPES buffer, $50 \mu \mathrm{mol} / 12-\mathrm{ME}, 100 \mathrm{U} / \mathrm{ml}$ penicillin, $100 \mathrm{mg} / \mathrm{ml}$ streptomycin, $2 \mathrm{mM}$ glutamine, $1 \mathrm{mmol} / 1$ sodium pyruvate and $20 \% \mathrm{FBS}$ ) at $37^{\circ} \mathrm{C}$ in a humidified incubator containing $5 \% \mathrm{CO}_{2}$ for 12 days. In the Flt $3 \mathrm{~L}$ group, the lobes were cultured with the aforementioned complete RPMI 640 media containing Flt3L (R\&D Systems Europe, Ltd., Abingdon, UK) at a concentration of $100 \mathrm{ng} / \mathrm{ml}$ for 12 days. The medium was replaced daily.

TDC isolation. Single cell suspensions were obtained by passing the disrupted thymic lobes from the 12-day-FTOC system through a 25-gauge hypodermic needle (BD Biosciences, Franklin Lakes, NJ, USA), and washed in PBS containing $1 \%$ FBS prior to use. A total of $1 \times 10^{6}$ thymocytes were incubated with rat anti-mouse $\mathrm{CD} 11 \mathrm{c}-\mathrm{PE}$ monoclonal antibodies (clone N418; $10 \mu 1 / 10^{7}$ cells; Miltenyi Biotec, Inc.) for $15 \mathrm{~min}$ at $4^{\circ} \mathrm{C}$. Following staining, the cells were washed once with PBS and incubated with anti-PE microbeads (Miltenyi Biotec, Inc.) for 
$15 \mathrm{~min}$ at $4^{\circ} \mathrm{C}$. The marked cells were washed, resuspended in PBS and purified using MACS. Subsequently, the CD11c ${ }^{+}$ thymocytes were resuspended in PBS containing 1\% FBS prior to staining. The CD11 $\mathrm{c}^{+}$thymocytes were separately incubated with monoclonal Bio-conjugated mouse B220 antibody (clone RA3-6B2; $10 \mu 1 / 10^{7}$ cells; Miltenyi Biotec, Inc.) for $\sim 20 \mathrm{~min}$ and strepavidin-microbeads (Miltenyi Biotec, Inc.) for $\sim 20 \mathrm{~min}$ at $4^{\circ} \mathrm{C}$. Finally, the $\mathrm{CD} 11 \mathrm{c}^{+} \mathrm{B} 220^{-}$ DCs (cDCs) and CD11c $\mathrm{c}^{+} 220^{+} \mathrm{DCs}(\mathrm{pDCs})$ were isolated using MACS.

Immunofluorescence staining and flow cytometric analysis. The thymic lobes from the 12-day-FTOC system were homogenized in PBS, and a suspension of thymocytes was derived by passing the sample through a cell filter. A total of $2 \times 10^{5}$ subsets of embryonic thymocyte cells were incubated with saturating quantities of fluorescein isothiocyanate (FITC)- and PE-labeled monoclonal antibodies for $30 \mathrm{~min}$ at $4^{\circ} \mathrm{C}$. The antibodies included anti-CD11c-FITC (clone N418), anti-CD4-PE (clone GK1.5), anti-CD8-PE (clone 53-6.7), anti-CD117-PE (clone 2B8; anti-Ia-PE (clone NIMR-4), anti-CD8-FITC (clone 53-6.7) and B220-biotin (clone RA3-6B2; all from eBioscience, San Diego, CA, USA), anti-CD11c PE (clone HL3) and anti-B220 FITC (clone RA3-6B2; BD Pharmingen, San Diego, CA, USA). Following staining, the cells were washed twice and resuspended in PBS for analysis. Subsequently, the cells were analyzed using a FACScalibur (BD Biosciences, Mountain View, CA, USA).

Optical microscopy. The thymic lobes from the 12-day-FTOC system were homogenized and sorted into $\operatorname{cDCs}\left(\sim 5 \times 10^{5}\right)$ and pDCs $\left(\sim 5 \times 10^{5}\right)$ using MACS. Subsequently, the cDCs and pDCs were stimulated in vitro with CpG2006 (GenScript, Nanjing, China) for $24 \mathrm{~h}$ at $37^{\circ} \mathrm{C}$, in a humidified incubator containing $5 \% \mathrm{CO}_{2}$. Following culture for $24 \mathrm{~h}$, the mature cDCs and pDCs stained with Giemsa (Sigma-Aldrich) were observed and images were captured under an optical microscope (Olympus CKX41; Olympus Corporation, Tokyo, Japan).

Mixed leukocyte cultures for assaying DC function. The culture system used in the present study has been described previously (19). Allogenic CD $4^{+}$T cells from the BALB/c mice were used as responders (14). Briefly, the adherent cells were initially removed by incubating splenic mononuclear cells (MNCs) at $37^{\circ} \mathrm{C}$ for $2 \mathrm{~h}$ in RPMI 1640 containing $10 \% \mathrm{FBS}$. The nonadherent splenic MNCs $\left(1 \times 10^{7}\right)$ were incubated with microbead-conjugated anti-mouse CD4 monoclonal antibody (clone L3T4; $10 \mu 1 / 10^{7}$ cells; Miltenyi Biotec $\mathrm{GmbH}$ ) and the $\mathrm{CD}^{+} \mathrm{T}$ cells were separated using MACS. The cDCs and pDCs from the FTOC system were used as stimulators. Graded doses of stimulator cells (between 100 and $3 \times 10^{4}$ cells) were added to the $\mathrm{T}$ cells $\left(3 \times 10^{5}\right)$ in a 96 -well round-bottom tissue-culture plate (Corning Incorporated, Corning, NY, USA). Following incubating with or without CpG2006 at $37^{\circ} \mathrm{C}$ for 4 days, the cell proliferation was determined using 3-(4,5-dimethyl thiazolyl-2)-2,5-diphenyltetrazolium bromide (Sigma-Aldrich). The resultant absorbance at $490 \mathrm{~nm}$ was read using a Dynatech MR7000 microtiter plate reader (Dynatech Laboratories, Chantilly, VA, USA).
Cytokine detection. The sorted cDCs $\left(3 \times 10^{5}\right)$ and $\mathrm{pDCs}\left(3 \times 10^{5}\right)$ were cultured with CpG2216 for $24 \mathrm{~h}$ to stimulate cytokine production. Following the $24 \mathrm{~h}$ culture, the supernatants were collected and quantified using an enzyme-linked immunosorbent assays (ELISA). The color development was measured at 490 nm using a Dynatech MR7000 microtiter plate reader. The mouse IL-12 p70 ELISA kit was purchased from BioSource International (Camarillo, CA, USA), whereas the mouse IFN- $\alpha$ ELISA kit was purchased from PBL Biomedical Laboratories (Piscataway, NJ, USA).

Reverse transcription-polymerase chain reaction (RT-PCR). Total RNA was extracted from $1 \times 10^{6}$ of the indicated cells using TRIzol reagent (Invitrogen Life Technologies), according to the manufacturer's instructions. First-strand complementary DNA (cDNA) was synthesized from $200 \mathrm{ng}$ total RNA in a $25 \mu \mathrm{l}$ reaction volume with the use of random primers (Takara Biotechnology Co., Ltd., Dalian, China) using Bio-Rad gradient PCR (Bio-Rad Laboratories, Inc., Hercules, CA, USA). Subsequently, the cDNA was amplified for 25 cycles consisting of $94^{\circ} \mathrm{C}$ for $30 \mathrm{sec}, 58^{\circ} \mathrm{C}$ for $30 \mathrm{sec}$ and $72^{\circ} \mathrm{C}$ for $45 \mathrm{sec}$, with a pair of oligonucleotide primers for the corresponding toll-like receptor (TLR). A glyceraldehyde-3-phosphate dehydrogenase (GAPDH) transcript was amplified in parallel as a control. The corresponding oligonucleotide primer sequences were as follows: TLR7, forward 5'-AGAGGCCCATGTGATCGTG-3' and reverse 5'-CGA GGGCAATTTCCACTTAGG-3'; TLR9, forward 5'-AAC ATGGTTCTCCGTCGAAGG-3' and reverse 5'-GTAGAT GCAGTTCCCGTCC-3'; and GAPDH, forward 5'-CCTAAG GCCAACCGTGAAAAG-3' and reverse 5'-TCTTCATGG TGCTAGGAGCCA-3'. The PCR products were fractionated on a $1.5 \%$ agarose gel (Sigma-Aldrich) and visualized using ethidium bromide $(0.5 \mu \mathrm{g} / \mathrm{ml}$; Sigma-Aldrich) staining.

Statistical analysis. All data are expressed as the mean \pm standard error of the mean. Student's $t$-test and a one-way analysis of variance were used to assess the difference between the two groups or among multiple groups, respectively. All data were analyzed using SPSS 16.0 (SPSS, Inc., Chicago, IL, USA). $\mathrm{P}<0.05$ was considered to indicate a statistically significant difference.

\section{Results}

$B M$-derived $C D 117^{+} H P C s$ differentiate into TDCs in the FTOC system in the presence of Flt3L. To determine the effect of Flt3L on the differentiation of HPCs into TDCs, BM CD117 $7^{+}$ HPCs were cultured using the FTOC system in the presence of Flt3L. The number of thymocytes from the Flt3L-treated group was twice the number observed in the control group. This suggested that Flt3L contributed to HPC proliferation in the FTOC system.

To further characterize the subset of proliferated thymic cells, different cell surface markers on the thymocytes were detected following 12 days of culture with or without Flt3L. Flow cytometric analysis revealed that the expression of CD11c in the Flt3L group was approximately nine times higher tha that in the control group (Fig. 1A). The proportion of CD8-CD11 $c^{+}$thymocytes increased in the presence of 
$\mathbf{A}$
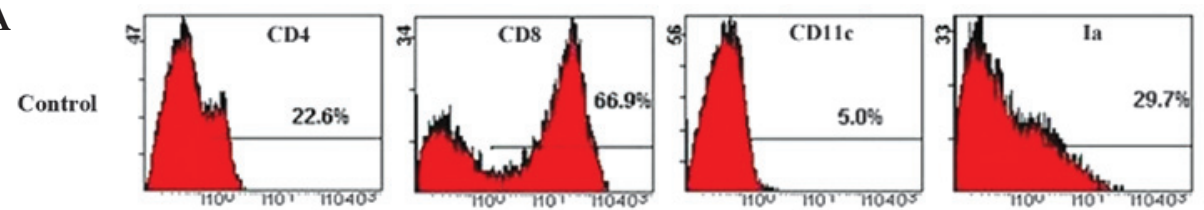

Flt3L
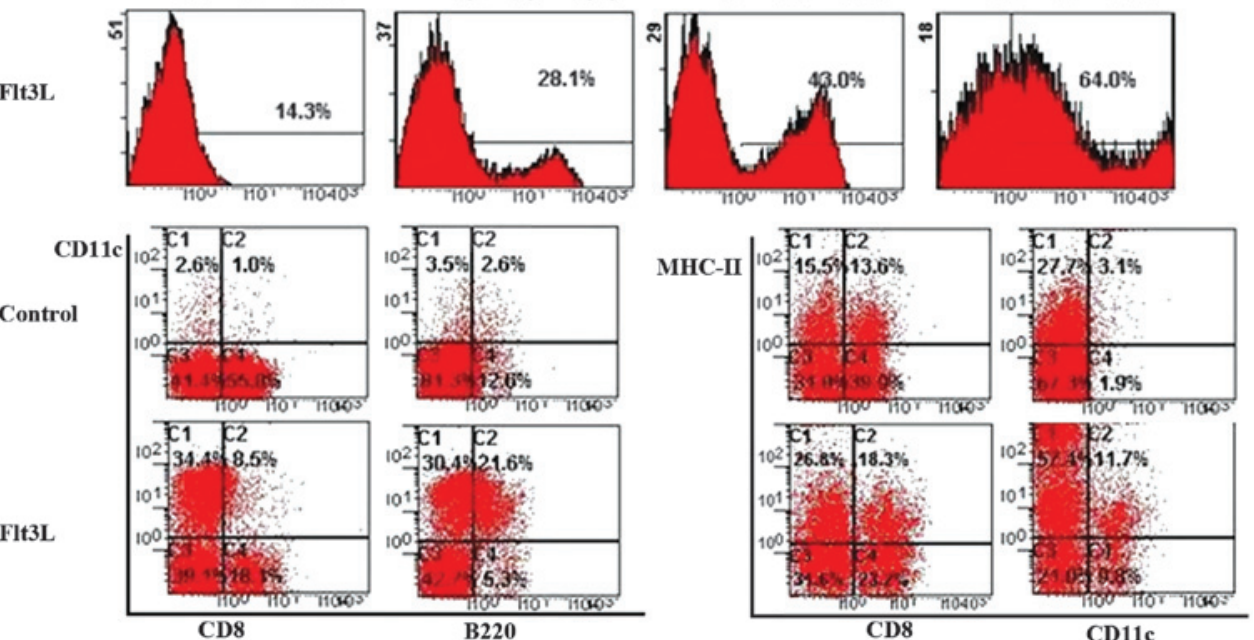

Figure 1. Phenotypic analysis of thymocytes recovered from FTOC, treated with or without Flt3L. Phenotype of the thymocytes following 12 days of culture in the absence or presence of Flt3L. Cells were recovered from fetal thymic lobes following 12 days of culture and were (A) single- or (B) double-labeled for flow cytometric analysis. A minimum of 10,000 cells were analyzed for the expression levels of CD4, CD8, CD11c, Ia and B220. The results are representative of a series of three independent experiments. The BM-derived CD117 $7^{+}$HPCs differentiated into TDCs in the FTOC system in the presence of Flt3L. FTOC, fetal thymus organ culture; Flt3L, Fms-like tyrosine kinase 3 ligand; MHC, major histocompatibility complex.

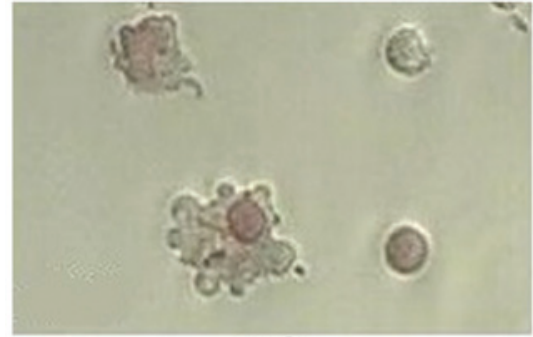

cDC

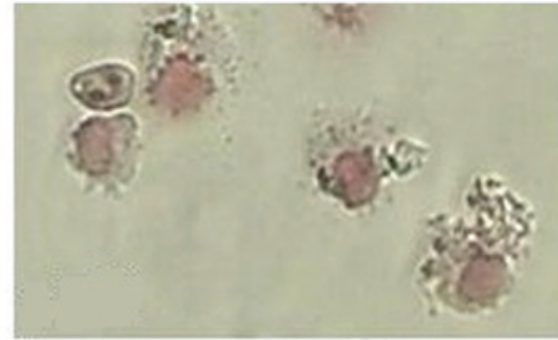

pDC

Figure 2. Morphology of thymic cDCs and pDCs. Giemsa staining was performed on the sorted thymic cDCs and pDCs from the Fms-like tyrosine kinase 3 ligand-treated fetal thymus organ culture, which were then cultured overnight with CpG2006. The results are representative of three independent experiments. (magnification, x200). The cDCs were large, cytoplasm-rich and irregular in shape with numerous membrane projections; pDCs exhibited a round shape with few membrane projections. cDC, conventional dendritic cell; pDC, plasmacytoid dendritic cell.

Flt3L (Fig. 1B). Similarly, the $\mathrm{Ia}^{+} \mathrm{CD} 11 \mathrm{c}^{+}$thymocytes from the Flt3L-treated FTOC proliferated faster than those in control cultures. In addition, the proportion of $\mathrm{cDCs}$ was also nine times higher in the Flt3L-treated group than in the control group, and the proportion of pDCs was eight times higher (Fig. 1B). These results confirmed the existence of a large number of TDCs (cDCs and $\mathrm{pDCs}$ ) in the Flt3L-treated group.

Morphological examination was performed to confirm the nature of the identified cells. Freshly isolated $\mathrm{CD} 11 \mathrm{c}^{+} \mathrm{B} 220^{+}$ pDCs from the FTOC system exhibited a round shape with few membrane projections, whereas the CD11c ${ }^{+} \mathrm{B} 220$-cDCs exhibited a large, cytoplasm-rich, irregular shape with numerous membrane projections. Followin overnught culture in the presence of CpG2006, these two types of TDCs were observed to exhibit a typical DC morphology with irregular membranes, bean-like nuclei and fine dendritic processes (Fig. 2). The results of the Giemsa staining indicated that the matured thymic cDCs and pDCs presented with the specific morphology of DCs.
TDCs from Flt3L-administered FTOC promote $\mathrm{CD}^{+} \mathrm{T}$ cell proliferation. To assess the functional properties of TDCs from Flt3L-treated FTOC, purified CD4 ${ }^{+} \mathrm{T}$ cells were used as responder cells to evaluate the allostimulatory capacity of the thymocytes generated in the 12-day Flt3L-treated FTOC system. As shown in Fig. 3, the CD11c ${ }^{+}$DCs were capable of stimulating the proliferation of allogeneic $\mathrm{T}$ cells with or without CpG2006 treatment. However, CD11 ${ }^{+}$DCs without Cp2006 treatment exhibited a relatively poor stimulatory capacity. This indicated that the thymocytes cultured in Flt3L-treated cultures were efficient antigen-presenting cells.

To further examine the potential of cDCs and pDCs in stimulating the proliferation of allogeneic $\mathrm{T}$ cells, mixed leukocyte cultures were performed. As shown in Fig. 4, the cDCs subjected to CpG2006 treatment proliferated faster than the pDCs. Therefore, cDCs and pDCs from the Flt3L-administered FTOC system exhibited different potentials in stimulating the proliferation of allogeneic $\mathrm{T}$ cells. 


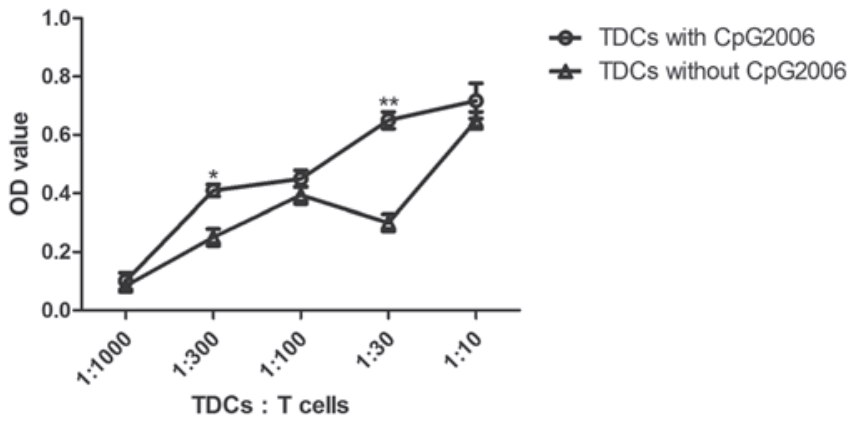

Figure 3. Mixed lymphocyte reaction-stimulatory capacity of TDCs generated using FTOC in the presence of Flt3L. TDCs derived from the 12-day Flt3L-treated FTOC were seeded in a series of dilutions as stimulators for allogeneic $\mathrm{T}$ cells $\left(3 \times 10^{5}\right)$ isolated from lymph nodes with or without CpG2006. After 5 days, the cultures were pulsed for $4 \mathrm{~h}$ with MTT. The resultant absorbance at $490 \mathrm{~nm}$ was read using an enzyme-linked immunosorbent assay instrument. The results are expressed as the mean of pooled data from three independent experiments, with five cultures at each dilution. ${ }^{*} \mathrm{P}<0.05$ and ${ }^{* * *} \mathrm{P}<0.01$, TDCs with CpG2006, vs. without CpG2006. TDCs, thymic dendritic cells; OD, optical density; FTOC, fetal thymus organ culture; Flt3L, Fms-like tyrosine kinase 3 ligand.

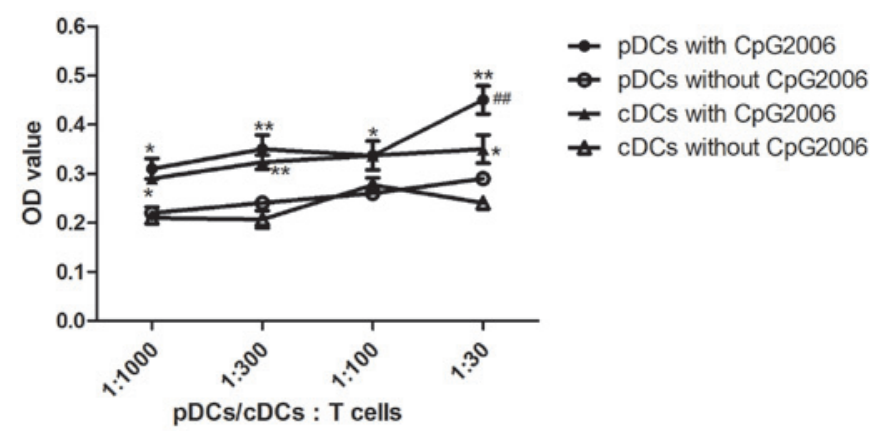

Figure 4. Allogenic mixed lymphocyte reaction. The allogenic mixed lymphocytes reaction was performed using purified $\mathrm{CD} 4^{+} \mathrm{T}$ cells $\left(3 \times 10^{5}\right.$ cells/well in 96-round-well plate) as responder cells. Thymic pDCs from Flt3L-treated FTOC with and without CpG2006 stimulation, and thymic cDCs from Flt3L-treatd FTOC with and without CpG2006, stimulation were examined. All the indicated cell subpopulations were purified or generated. The results are representative of three independent experiments. ${ }^{*} \mathrm{P}<0.05$ and ${ }^{* *} \mathrm{P}<0.01$, pDCs with CpG2006, vs. pDCs without $\mathrm{CpG} 2006$; ${ }^{*} \mathrm{P}<0.05$ and ${ }^{* *} \mathrm{P}<0.01$, cDCs with CpG2006, vs. cDCs without CpG2006. ${ }^{\# \#} \mathrm{P}<0.01$, pDCs with CpG2006, vs. cDCs with CpG2006. FTOC, fetal thymus organ culture; Flt3L, Fms-like tyrosine kinase 3 ligand; cDC, conventional dendritic cell; pDC, plasmacytoid dendritic cell; OD, optical density.

Cytokine production by thymic cDCs and pDCs. The production of particular cytokines is an indication of the intrathymic role of TDCs, aside from antigen presentation. However, only a small number of cytokines produced by TDCs in situ in a steady state have been observed until now. In the present study, thymic cDCs and pDCs were stimulated with $\mathrm{CpG}$ oligonucleotides (CpG2216) to assess their cytokine secretion capacities. As shown in Fig. 5, the cDCs secreted a low level of IL-12 and IFN- $\alpha$, whereas the pDCs secreted substantial quantities of IFN- $\alpha$, but not IL-12. This indicated that the thymic pDCs and cDCs exhibited diverse capacities in IFN- $\alpha$ and IL-12 production.

In addition, RT-PCR was applied to assess the expression of levels of TLR7 and TLR9 in the thymic cDCs and pDCs stimulated by $\mathrm{CpG} 2216$. It was found that the thymic pDCs, but not the cDCs, expressed high levels of TLR7 and TLR9

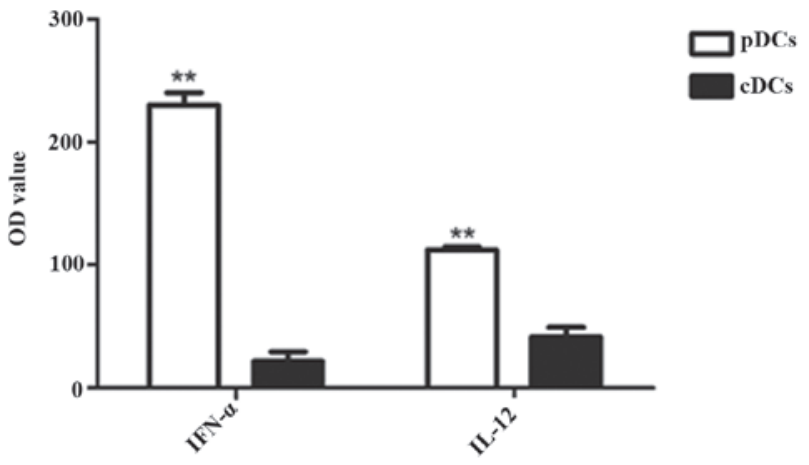

Figure 5. Comparison of cytokine production between thymic pDCs and cDCs following CpG2216 stimulation. Thymic cDCs and pDCs were purified and were stimulated with CpG2216 overnight. Following 24 h culture, the supernatants were assayed for IL-12 and IFN- $\alpha$ using enzyme-linked immunosorbent assays. Comparison of cytokine production between the thymic pDCs and cDCs following CpG2216 stimulation is shown $\left({ }^{* *} \mathrm{P}<0.01\right.$, compared with the $\mathrm{cDCs}$ ). Results are representative of thee independent experiments and are expressed as the mean \pm standard error of the mean. IL-12, interleukin-12; IFN- $\alpha$, interferon- $\alpha$; cDC, conventional dendritic cell; pDC, plasmacytoid dendritic cell; OD, optical density.

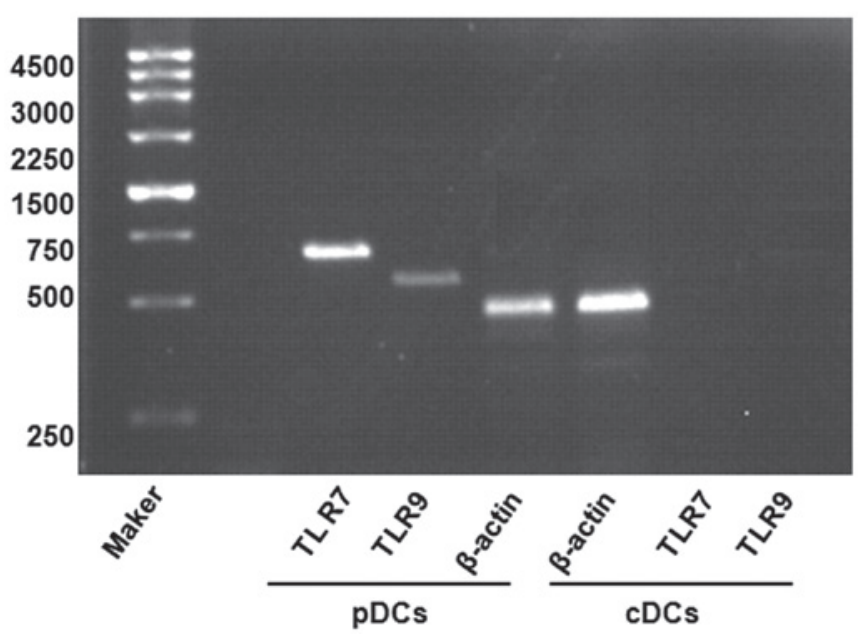

Figure 6. TLR profiles of thymic cDCs and pDCs. The expression of TLR7, TLR9 and GAPDH in the thymic pDCs and cDCs from Fms-like tyrosine kinase 3 ligand-treated fetal thymus organ culture were purified. The expression of GAPDH, TLR7 and TLR9 in the thymic pDCs are shown on the left; expression of GAPDH, TLR7 and TLR9 in the thymic cDCs are shown on the right. Total RNAs were extracted from $1 \times 10^{6}$ of the indicated cells. Reverse transcription-polymerase chain reaction was performed. The results are representative of three independent experiments. $\mathrm{cDC}$, conventional dendritic cell; pDC, plasmacytoid dendritic cell; TLR, toll-like receptor; GAPDH, glyceraldehyde-3-phosphate dehydrogenase.

(Fig. 6). These results are consistent with those of previous studies (20). Therefore, the thymic cDCs and pDCs from the culture system presented with functional capacities in cytokine production.

\section{Discussion}

DCs are critical in immunotherapy against infections. However, the limited availability of DCs is the predominant restriction in biological or clinical investigations. Several reports have used in vitro differentiated myeloid-derived DCs (21), which are induced principally by granulocyte-macrophage 
colony-stimulating factor (GM-CSF) in combination with other cytokines, including tumor necrosis factor- $\alpha$, IL-4 and stem cell factor $(16,22,23)$. Flt3L and GM-CSF are important cytokines during DC development in a steady state, and the two are expressed in DC progenitors (24). Notably, GM-CSF is expressed predominantly in monocytes, cDCs and skin DCs, whereas Flt3 is expressed in cDCs and pDCs (25). In the thymus, Flt3 is expressed by a subset of early $\mathrm{T}$ lineage progenitors (ETPs), and these Flt $3^{+}$ETPs represent the earliest intrathymic $\mathrm{T}$ lineage progenitors identified thus far (26) In addition, HPCs exhibit different mobilization kinetics in response to Flt3L, resulting in the preferential mobilization of HPCs at day 5 followed by HPC mobilization at day 10 (27). Although DCs are traditionally generated in vitro with cytokine cocktails containing GM-CSF, with or without IL-4, the in vivo expansion of DC populations is usually accomplished through the administration of the Flt3 $\mathrm{L}$ growth factor $(3,4)$. Additionally, DCs can arise from lymphoid- and myeloid-committed BM precursors, which lack markers of differentiated immune lineage cells and express the Flt3 receptor (lin-flt $\left.3^{+}\right)$(28). Few studies have used Flt3L in DC expansion in vitro.

In the present study, comprehensive analysis of the role of Flt3L on the development of thymic DCs (cDCs and pDCs) from BM-derived HPCs was performed. In order to remove pre-thymocytes in the embryonic thymic lobes, freshly collected thymic lobes were treated with 2 -dGuo prior to seeding the BM-derived HPCs within a hanging drop culture system. In the Flt3L-FTOC system, two distinct DC subsets (cDCs and $\mathrm{pDCs}$ ) were characterized from the BM-derived HPCs, which were separately identified as CD11 ${ }^{+} \mathrm{B} 220^{-}$and $\mathrm{CD} 11 \mathrm{c}^{+} \mathrm{B} 220^{+}$in the mouse thymus. Notably, Flt3L treatment was capable of expanding cDCs and pDCs, particularly, pDCs. Following overnight culture in the presence of CpG2006, the cDCs and pDCs generated by the Flt3L-FTOC system exhibited a typical DC morphology with irregular membranes, bean-like nuclei and fine dendritic projections. Functionally, these TDCs (cDCs and pDCs) from the Flt3L-FTOC system stimulated allogenic $\mathrm{T}$ cell proliferation.

The present study also observed that the pDCs, but not the cDCs, from the Flt3L-FTOC system expressed high levels of TLR7 and TLR9. This suggested that thymic pDCs are a major component of TLR7- and TLR9-dependent inflammation. The TLR7/9-dependent pathway appears to be a predominant mode of nucleic acid sensing in pDCs, although additional DNA sensors, including DHX9/DHX36 have been suggested (29). pDCs are innate immune cells, which circulate in the blood and lymphoid tissues and, upon stimulation by unmethylated CpG DNA through the engagement of TLR7 and TLR9, pDCs can be specialized to produce substantial quantities of IFN- $\alpha(30,31)$. The present study also observed that these pDCs secreted higher levels of IL-12 and IFN- $\alpha$, compared with the cDCs following stimulation with $\mathrm{CpG} 2216$. These findings suggested that pDCs are more important in protecting the thymus against viral infection than cDCs.

In conclusion, the current limitations in isolating large numbers of DCs has been the predominant obstacle in investigating the biological functions and clinical applications of DCs. The present study demonstrated that the Flt3L-FTOC system was able to support in vitro expansion of a novel TDC lineage in culture from a number of $\mathrm{CD}_{117^{+}} \mathrm{HPCs}$. Notably, this culture system provided the distinct expansion of thymic pDCs, however, it was not able to produce pure cDCs or pDCs. Therefore, this culture system requires improvement to produce a single subset of DCs. These findings have important implications for the current opinion that in vitro-generated pDCs may offer potential in translational medicine.

\section{Acknowledgements}

This study was supported by the National Natural Science Foundation of China Grants (grant nos. 30400395 and 31500718) and the Projects of Soochow Science And Technology Plans (grant no. SYS201438).

\section{References}

1. Lee HK and Iwasaki A: Innate control of adaptive immunity: Dendritic cells and beyond. Semin Immunol 19: 48-55, 2007.

2. Shortman K and Naik SH: Steady-state and inflammatory dendritic-cell development. Nat Rev Immunol 7: 19-30, 2007.

3. Gilliet M, Boonstra A, Paturel C, Antonenko S, Xu XL, Trinchieri G, O'Garra A and Liu YJ: The development of murine plasmacytoid dendritic cell precursors is differentially regulated by FLT3-ligand and granulocyte/macrophage colony-stimulating factor. J Exp Med 195: 953-958, 2002.

4. Miller G, Pillarisetty VG, Shah AB, Lahrs S and DeMatteo RP: Murine Flt3 ligand expands distinct dendritic cells with both tolerogenic and immunogenic properties. J Immunol 170: 3554-3564, 2003.

5. Luche H, Ardouin L, Teo P, See P, Henri S, Merad M, Ginhoux F and Malissen B: The earliest intrathymic precursors of CD8alpha (+) thymic dendritic cells correspond to myeloid-type double-negative 1c cells. Eur J Immunol 41: 2165-2175, 2011.

6. Ardavin C, Wu L, Li CL and Shortman K: Thymic dendritic cells and $\mathrm{T}$ cells develop simultaneously in the thymus from a common precursor population. Nature 362: 761-763, 1993.

7. Kadowaki N, Antonenko S and Liu YJ: Distinct CpG DNA and polyinosinic-polycytidylic acid double-stranded RNA, respectively, stimulate CD11c-type 2 dendritic cell precursors and CD11c+ dendritic cells to produce type I IFN. J Immunol 166: 2291-2295, 2001.

8. Diebold SS, Kaisho T, Hemmi H, Akira S and Reis e Sousa C: Innate antiviral responses by means of TLR7-mediated recognition of single-stranded RNA. Science 303: 1529-1531, 2004.

9. Jarrossay D, Napolitani G, Colonna M, Sallusto F and Lanzavecchia A: Specialization and complementarity in microbial molecule recognition by human myeloid and plasmacytoid dendritic cells. Eur J Immunol 31: 3388-3393, 2001.

10. Bendriss-Vermare N, Barthélémy C, Durand I, Bruand C, Dezutter-Dambuyant C, Moulian N, Berrih-Aknin S, Caux $\mathrm{C}$, Trinchieri $\mathrm{G}$ and Brière $\mathrm{F}$ : Human thymus contains IFN-alpha-producing CD11c (-), myeloid CD11c (+) and mature interdigitating dendritic cells. J Clin Invest 107: 835-844, 2001.

11. Gurney KB, Colantonio AD, Blom B, Spits $\mathrm{H}$ and Uittenbogaart $\mathrm{CH}$ : Endogenous IFN-alpha production by plasmacytoid dendritic cells exerts an antiviral effect on thymic HIV-1 infection. J Immunol 173: 7269-7276, 2004.

12. Fohrer H, Audit IM, Sainz A, Schmitt C, Dezutter-Dambuyant C and Dalloul AH: Analysis of transcription factors in thymic and CD34+ progenitor-derived plasmacytoid and myeloid dendritic cells: Evidence for distinct expression profiles. Exp Hematol 32: 104-112, 2004.

13. Keir ME, Stoddart CA, Linquist-Stepps V, Moreno ME and McCune JM: IFN-alpha secretion by type 2 predendritic cells up-regulates MHC class I in the HIV-1-infected thymus. J Immunol 168: 325-331, 2002.

14. Varas A, Vicente A, Sacedón R and Zapata AG: Interleukin-7 influences the development of thymic dendritic cells. Blood 92: 93-100, 1998. 
15. Adolfsson J, Borge OJ, Bryder D, Theilgaard-Mönch K, Astrand-Grundström I, Sitnicka E, Sasaki Y and Jacobsen SE: Upregulation of Flt3 expression within the bone marrow Lin (-) Sca1 (+) c-kit (+) stem cell compartment is accompanied by loss of self-renewal capacity. Immunity 15: 659-669, 2001.

16. Maraskovsky E, Brasel K, Teepe M, Roux ER, Lyman SD, Shortman K and McKenna HJ: Dramatic increase in the numbers of functionally mature dendritic cells in Flt3 ligand-treated mice: Multiple dendritic cell subpopulations identified. J Exp Med 184: 1953-1962, 1996

17. O'Keeffe M, Hochrein H, Vremec D, Pooley J, Evans R, Woulfe S and Shortman K: Effects of administration of progenipoietin 1, Flt-3 ligand, granulocyte colony-stimulating factor and pegylated granulocyte-macrophage colony-stimulating factor on dendritic cell subsets in mice. Blood 99: 2122-2130, 2002.

18. Chen W, Antonenko S, Sederstrom JM, Liang X, Chan AS, Kanzler H, Blom B, Blazar BR and Liu YJ: Thrombopoietin cooperates with FLT3-ligand in the generation of plasmacytoid dendritic cell precursors from human hematopoietic progenitors. Blood 103: 2547-2553, 2004.

19. Suss G and Shortman K: A subclass of dendritic cells kills CD4 T cells via Fas/Fas-ligand-induced apoptosis. J Exp Med 183: 1789-1796, 1996.

20. Ganguly D, Chamilos G, Lande R, Gregorio J, Meller S, Facchinetti V, Homey B, Barrat FJ, Zal T and Gilliet M: Self-RNA-antimicrobial peptide complexes activate human dendritic cells through TLR7 and TLR8. J Exp Med 206: 1983-1994, 2009.

21. Cella M, Sallusto F and Lanzavecchia A: Origin, maturation and antigen presenting function of dendritic cells. Curr Opin Immunol 9: 10-16, 1997.

22. Romani N, Reider D, Heuer M, Ebner S, Kämpgen E, Eibl B Niederwieser D and Schuler G: Generation of mature dendritic cells from human blood. An improved method with special regard to clinical applicability. J Immunol Methods 196: $137-151,1996$
23. Zhou LJ and Tedder TF: CD14+ blood monocytes can differentiate into functionally mature CD83+ dendritic cells. Proc Natl Acad Sci USA 93: 2588-2592, 1996.

24. Kingston D, Schmid MA, Onai N, Obata-Onai A, Baumjohann D and Manz MG: The concerted action of GM-CSF and Flt3-ligand on in vivo dendritic cell homeostasis. Blood 114: 835-843, 2009.

25. McKenna HJ, Stocking KL, Miller RE, Brasel K, De Smedt T, Maraskovsky E, Maliszewski CR, Lynch DH, Smith J, Pulendran B, et al: Mice lacking flt3 ligand have deficient hematopoiesis affecting hematopoietic progenitor cells, dendritic cells and natural killer cells. Blood 95: 3489-3497, 2000.

26. Sambandam A, Maillard I, Zediak VP, Xu L, Gerstein RM, Aster JC, Pear WS and Bhandoola A: Notch signaling controls the generation and differentiation of early $\mathrm{T}$ lineage progenitors. Nat Immunol 6: 663-670, 2005.

27. de Kruijf EJ, Hagoort H, Velders GA, Fibbe WE and van Pel M: Hematopoietic stem and progenitor cells are differentially mobilized depending on the duration of Flt3-ligand administration. Haematologica 95: 1061-1067, 2010.

28. Karsunky H, Merad M, Cozzio A, Weissman IL and Manz MG Flt3 ligand regulates dendritic cell development from Flt3+ lymphoid and myeloid-committed progenitors to Flt3+ dendritic cells in vivo. J Exp Med 198: 305-313, 2003.

29. Kim T, Pazhoor S, Bao M, Zhang Z, Hanabuchi S, Facchinetti V, Bover L, Plumas J, Chaperot L, Qin J and Liu YJ: Aspartate-g lutamate-alanine-histidine box motif (DEAH)/RNA helicase A helicases sense microbial DNA in human plasmacytoid dendritic cells. Proc Natl Acad Sci USA 107: 15181-15186, 2010.

30. Beignon AS, McKenna K, Skoberne M, Manches O, DaSilva I, Kavanagh DG, Larsson M, Gorelick RJ, Lifson JD and Bhardwaj N: Endocytosis of HIV-1 activates plasmacytoid dendritic cells via Toll-like receptor-viral RNA interactions. J Clin Invest 115: 3265-3275, 2005 .

31. Heil F, Hemmi H, Hochrein H, Ampenberger F, Kirschning C, Akira S, Lipford G, Wagner H and Bauer S: Species-specific recognition of single-stranded RNA via toll-like receptor 7 and 8 . Science 303: 1526-1529, 2004. 